\title{
Increasing number of hospital beds has inconsistent effects on delaying bed shortages due to COVID-19
}

\author{
Miles Roberts*, Helena Duplechin Seymour ${ }^{\dagger}$ \\ Faculty Advisor: Alexander Dimitrov ${ }^{\ddagger}$
}

June 1st 2021

\section{Abstract}

SARS-CoV-2, the virus responsible for COVID-19, has killed hundreds of thousands of Americans. Physical distancing measures and record-setting vaccine roll out played a key role in slowing COVID-19 spread, but the advent of new SARS-CoV-2 variants remains a real threat. Implementing strategies to minimize COVID-19 hospitalizations will be key to controlling the toll of COVID-19 variants and future novel pathogens, but non-physical distancing strategies receive relatively little attention. We present a novel system of differential equations designed to predict the relative effects of hospitalizing fewer COVID-19 patients and increasing ICU bed availability on delaying ICU bed shortages. This model, which we call SEAQIRD, was calibrated to mortality data on two US states with different peak infection times from mid-March - mid-May 2020. It found that when the probability of hospitalization is already low, decreasing it further can have a large effect on delaying an ICU bed shortage in both states. Meanwhile, altering the proportion of ICU beds available to COVID-19 patients had markedly different effects on when a bed shortage was reached in the two states. This trend remained consistent when the model's most sensitive parameters were altered.

\section{Introduction}

At the time of writing this, John's Hopkins University reports that about 600,000 Americans have died of COVID-19 (https://coronavirus.jhu.edu/) - the disease caused by the coronavirus SARS-CoV-2. Ever since the initial outbreak,

\footnotetext{
*Michigan State University, 360.335.7240, robe1195@msu.edu

†Washington State University Vancouver, 850.508.1806, helena.coker@wsu.edu

${ }^{\ddagger}$ Washington State University Vancouver, 360.546.9385, alex.dimitrov@wsu.edu
} 
the primary goal of US public health measures has been to slow COVID-19 infection rates enough that hospitals are not overwhelmed by a large influx of diseased individuals, also phrased as "flattening the curve". Until the recent vaccine roll-out, this goal was mostly achieved through physical distancing any measure that increases the average distance or number of barriers between individuals from different households. Physical distancing is often defined by the closing of workplaces, schools, and non-essential businesses [34], but the wearing of personal protective equipment (PPE) like gloves, face-coverings, and eye protection also match this broad definition. Physical distancing measures have been invaluable for mitigating COVID-19 spread, lowering the growth rate of the disease [9] and preventing at least half a million COVID-19 cases [34]. However, physical distancing is not without drawbacks.

Physical distancing imposes significant economic and psychological costs that are themselves the common subject of COVID-19 models [29]. Closing businesses and restricting travel in response to the COVID-19 pandemic disrupted US supply chains [14], exacerbated social inequalities [11], and increased rates of anxiety and depression [6]. There's even evidence to suggest that physical or social distancing may increase the spread of conspiracy theories [13]. Furthermore, physical distancing measures are ultimately voluntary and experts expect such measures to be less effective as lockdowns continue and isolated individuals inevitably grow bored [24]. News outlets report numerous stories of Americans resisting physical distancing measures, especially the wearing of masks [8]. Physical distancing could be the best tool America has for halting the spread of future COVID-19 variants but considering supplementary strategies may limit the need for physical distancing, and therefore its consequences, in future pandemics.

We present a model that considers two non-physical distancing strategies to prevent overwhelming hospitals with COVID-19 infections: (1) hospitalizing fewer patients with COVID-19 and (2) increasing hospital capacity for COVID19 patients. The former can be accomplished by selectively hospitalizing only serious COVID-19 cases, allowing less serious cases to self-isolate, while the latter can be accomplished by increasing the number of ICU beds, ventilators, PPE, and hospital staff. For simplicity, we focus on just ICU bed counts as a measure of hospital capacity and only consider the states of Washington (WA) and Colorado $(\mathrm{CO})$ because of their similar case numbers but different peak infection times from March - May 2020.

Washington was the first state in the U.S. to have confirmed COVID-19 infections [28]. When COVID-19 first arrived there, it spread rapidly among the elderly and nurses at long-term care facilities [25]. Newspapers at the time reported a reasonable fear that there would not be enough hospital beds in the whole state to care for the coming wave of COVID-19 infected patients [4]. WA enacted stay-at-home orders in mid-March and many other states like $\mathrm{CO}$ soon followed, as uncontrolled community-level transmission was already occurring in other states [23]. In fact, early in the pandemic, CO had one of the highest death counts in the US, just behind California [30]. Thanks to policies to expand hospital capacity and enact physical distancing, Washington 
and $\mathrm{CO}$ avoided exceeding the state-wide limit on hospital beds from March - May. Thus, knowing the relative benefits of increasing hospital capacity vs decreasing hospitalization in either of these states is still relevant today. Neither of these strategies should replace physical distancing, of course, but they can still contribute to mitigation efforts.

\section{Methods}

We employed a system of ordinary differential equations to model the spread of COVID-19 in WA and CO. We chose to model just WA and CO because of their similar case numbers during their initial outbreaks, their high-quality reporting according to COVID-tracking project (see Table S1), but different peak infection times. We further focused on data from only the period of March 15th - May 18th as this captured the majority of the first "infection wave" in both states. Focusing on this single period allowed us to avoid explicitly modeling complicated changes in human behavior that eventually lead to multiple waves of infection in both states. This focus also makes our study more relevant to future initial pandemic scenarios where public health agencies have relatively little data on disease dynamics. Most of the model parameters were estimated from previous studies on COVID-19 spread. The remaining parameters were estimated by minimizing the sum of squared errors between the model and data on cumulative deaths over time. Our model was calibrated to mortality data only because, due to testing limitations, counts of COVID-19 deaths are generally considered more reliable than counts of COVID-19 cases [3]. All simulations and analyses were conducted in R [31]. Simulation, optimization, and data visualization were all executed with the deSolve, Flexible Modeling Environment (FME), and ggplot2 packages respectively [35],[36],[41].

\subsection{Gathering Data}

All of the datasets input into our model are listed in Table S2, along with hyperlinks to their associated websites. Counts of COVID-19 deaths during the period of March 15th - May 18th were downloaded from the novel coronavirus infection map hosted by the University of Washington. Counts of COVID-19 hospitalizations were downloaded from a publicly available database hosted by Definitive Healthcare, a healthcare data analytics company (https://www.definitivehc.com/about). To correct for under-reporting of hospitalizations, data on hospitalization response rates were downloaded from each state's respective Department of Health $(\mathrm{DOH})$ website. This data has since been removed from the WA DOH website, but a copy of the dataset is included in the supplemental (Table S3). Next, the number of ICU beds in WA was downloaded directly from the WA DOH website, but this information could not be found on the $\mathrm{CO}$ DOH website. Thus, a report of 2018 ICU bed counts from a local CO news organization was used instead. Finally, population size estimates for both states were acquired from their respective US Census Bureau websites. 


\subsection{Correction for under-reporting by hospitals}

Not all hospitals report counts of COVID-19 cases to their state governments every day. For the states we studied, fewer hospitals reported COVID-19 hospitalizations during weekends than weekdays (see Tables S3 and S4). The lack of daily reporting partly contributes to hospitalization reports underestimating the true number of hospitalizations. To account for under-reporting of hospitalizations, we applied a simple correction based on the assumption that the ratio of hospitalizations to number of hospitals was the same for the sample of reporting hospitals in a state as it is for all hospitals in a state. In other words:

$$
\frac{U}{K}=\frac{u}{k}
$$

Where $\mathrm{U}$ is the number of hospitalizations in the entire state, $\mathrm{K}$ is the number of hospitals in the entire state, $u$ is the number of reported hospitalizations, and $\mathrm{k}$ is number of reporting hospitals. $\mathrm{U}$ is the only unknown in this equation, meaning it can be calculated as:

$$
U=\frac{K u}{k}
$$

To apply this correction to the entire time series, we wrote $\mathrm{U}$ and $\mathrm{u}$ as functions of time $\mathrm{U}(\mathrm{t})$ and $\mathrm{u}(\mathrm{t})$ respectively:

$$
U(t)=\frac{K u(t)}{k}
$$

The WA DOH only counted the number of reporting hospitals for May 9th - May 15th, so data from this period was extrapolated to the entire time span of March 15th - May 18th (see Table S3). There are 92 acute care hospitals in the entire state, but only 66 hospitals reported case counts every day for May 9 th - May 15 th on average. Thus:

$$
U(t)=\frac{92 u(t)}{66}
$$

The CO DOH website also reports the percentage of hospitals that update COVID-19 data. However, these data are deleted from the website weekly. Thus, the average percentage of hospitals reporting data had to be estimated from data from July 4th - July 9th (see Table S4). About 75.43 percent of CO hospitals reported COVID-19 cases every day during this period on average. Thus, the applied correction was:

$$
U(t)=\frac{100 u(t)}{75.43}
$$

\subsection{Model formulation}

Our system of ordinary differential equations included a total of 7 state variables and 8 parameters, which are defined in Tables 1 and diagrammed in Figure 1. 


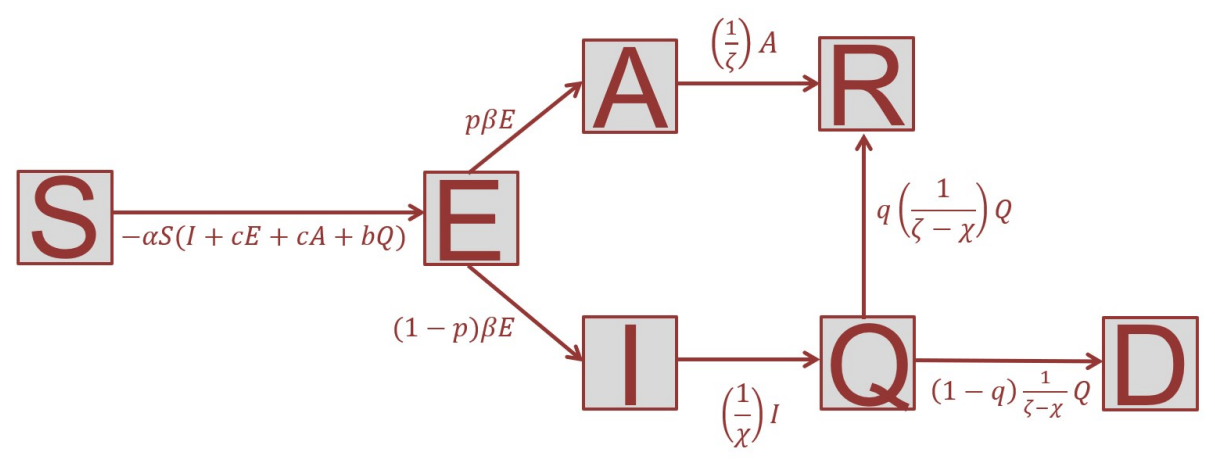

Figure 1: A compartment diagram depicting the system of ordinary differential equations used in this study. See Tables S5 and S6 for variable and parameter definitions.

From Figure 1, the full set of differential equations describing the spread of COVID-19 in our model can be written as:

$$
\begin{gathered}
\frac{d S}{d t}=-\alpha S(I+c E+c A+b Q) \\
\frac{d E}{d t}=\alpha S(I+c E+c A+b Q)-\beta E \\
\frac{d A}{d t}=p \beta E-\left(\frac{1}{\zeta}\right) A \\
\frac{d I}{d t}=(1-p) \beta E-\frac{1}{\chi} I \\
\frac{d Q}{d t}=\frac{1}{\chi} I-\frac{1}{\zeta-\chi} Q \\
\frac{d R}{d t}=q \frac{1}{\zeta-\chi} Q+\frac{1}{\zeta} A \\
\frac{d D}{d t}=(1-q) \frac{1}{\zeta-\chi} Q
\end{gathered}
$$

We lastly define the number of hospitalized individuals at any moment in time $H(t)$ as:

$$
H(t)=h Q
$$

Where $h \in[0,1]$. In other words, the number of hospitalized individuals is assumed to be a constant proportion of the total number of quarantined individuals, which includes both hospitalized and home-bound individuals. 
Table 1: Definitions of parameters and state variables in SEAQIRD model.

\begin{tabular}{|c|c|c|c|}
\hline Variable & Definition & Value & Reference \\
\hline$\alpha$ & $\begin{array}{l}\text { Contact rate between sus- } \\
\text { ceptible and infected }\end{array}$ & fitted & NA \\
\hline$\beta$ & $\begin{array}{l}\text { inverse of incubation pe- } \\
\text { riod }\end{array}$ & $1 / 3.60$ days & Li et al. 2020 \\
\hline$\zeta$ & recovery time & 24.7 days & $\begin{array}{l}\text { Verity et al. } \\
2020\end{array}$ \\
\hline$\chi$ & $\begin{array}{l}\text { time until self-isolation } \\
\text { once symptoms appear }\end{array}$ & 1 day & NA \\
\hline$p$ & $\begin{array}{l}\text { probability of asymp- } \\
\text { tomatic infection, given } \\
\text { exposure }\end{array}$ & 0.86 or 0.425 & $\begin{array}{l}\text { Li et al. } \\
2020 ; \\
\text { Lavezzo } \\
\text { et al. } 2020\end{array}$ \\
\hline$q$ & $\begin{array}{l}\text { probability of surviving a } \\
\text { symptomatic infection }\end{array}$ & 0.986 & $\begin{array}{l}\text { Wu et al. } \\
2020\end{array}$ \\
\hline$b$ & $\begin{array}{l}\text { infectiveness of quaran- } \\
\text { tined individuals relative } \\
\text { to non-quarantined in- } \\
\text { fected individuals }\end{array}$ & 0.1 & NA \\
\hline$c$ & $\begin{array}{l}\text { infectiveness of exposed } \\
\text { and asympotmatic indi- } \\
\text { viduals relative to non- } \\
\text { quarantined infected indi- } \\
\text { viduals }\end{array}$ & 0.55 & Li et al. 2020 \\
\hline$S$ & $\begin{array}{l}\text { number of susceptible in- } \\
\text { dividuals }\end{array}$ & NA & NA \\
\hline$E$ & $\begin{array}{l}\text { number of exposed, but } \\
\text { not infectious individuals }\end{array}$ & NA & NA \\
\hline$A$ & $\begin{array}{l}\text { number of infected, but } \\
\text { asymptomatic individuals }\end{array}$ & NA & NA \\
\hline$Q$ & $\begin{array}{l}\text { number of infected, symp- } \\
\text { tomatic, and quarantined } \\
\text { individuals }\end{array}$ & NA & NA \\
\hline$I$ & $\begin{array}{l}\text { number of infected, } \\
\text { symptomatic, and non- } \\
\text { quarantined individuals }\end{array}$ & NA & NA \\
\hline$R$ & $\begin{array}{l}\text { number of recovered indi- } \\
\text { viduals }\end{array}$ & NA & NA \\
\hline$D$ & $\begin{array}{l}\text { number of dead individu- } \\
\text { als }\end{array}$ & NA & NA \\
\hline
\end{tabular}

Equations 1 through 7 will be referred to as the SEAQIRD model for the 
remainder of the paper. The SEAQIRD model begins with assuming that susceptible individuals are exposed to COVID-19 at rates proportional to how often they contact individuals harboring SARS-CoV-2 $(\alpha)$. These individuals fall into multiple categories: symptomatic infected (I), exposed but undetermined symptom development (E), asymptomatic infected (A), and symptomatic isolated infected (Q, for quarantined, which can mean either "home-bound" or "hospitalized"). The scaling factors $\mathrm{c}$ and $\mathrm{b}$ are meant to reflect the "infectiveness" of individuals in the $\mathrm{E}, \mathrm{A}$, and $\mathrm{Q}$ compartments relative to the I compartment. We chose $c$ to be 0.55 , reflecting the finding that asymptomatic individuals are 55 percent as infectious as symptomatic individuals [19]. We assumed $b$ to be 0.1 , reflecting how isolated individuals ideally contact susceptible individuals only rarely.

Once an individual is exposed (E) to SARS-CoV2, they were assumed to have a probability $p$ of becoming asymptomatically infected (A) and a probability of $1-p$ of becoming symptomatically infected (I). Furthermore, the rate at which $\mathrm{E}$ individuals transformed into $\mathrm{A}$ or I individuals was assumed to be proportional the inverse of the incubation period, $\beta$, estimated as 1/6.6 days [19]. Asymptomatic individuals were assumed to never perish from COVID-19 and to recover from the disease at a rate proportional to $1 / \zeta$, the inverse of the recovery period. The recovery period, $\zeta$, was estimated from a previous study on COVID-19 severity as 24.7 days [39]. This result agrees with other studies on COVID-19 suggesting that infected asymptomatic individuals typically shed SARS-CoV-2 viruses 15 - 26 days after initial infection [21].

Once an exposed individual (E) individual develops into a symptomatically infected individual (I), they mix with the population for $\chi$ days before isolating themselves (see equations 5 and 4 ). $\chi$ was assumed to be 1 day in all SEAQIRD models. Once isolated, the infectiveness of symptomatic individuals was assumed to drop to $0.1 \alpha$. This rate was intentionally made non-zero to account for any minor contact isolated individuals have with susceptible individuals, since quarantine measures are rarely perfect. Once isolated $(\mathrm{Q})$, individuals had a probability $q$ of recovering from the disease and a probability $1-q$ of dying (see equations 6 and 7). The value of q was estimated as 0.986, again based on COVID-19 studies in China [42]. In addition, some of the rate constants in equations 6 and 7 were assumed to be the inverse of recovery period $-\chi$, where the " $\chi$ " accounts for the time symptomatic individuals spend mixing with the population before isolating themselves.

For the sake of simplicity, we assume that recovered individuals $(\mathrm{R})$ are not susceptible to re-infection with SARS-CoV-2. This is not known to be strictly true for SARS-CoV-2 infection in humans, but it is reasonable given how nonhuman primates respond to SARS-CoV-2 infection [7].

\subsection{Choosing initial conditions}

Given that SARS-CoV-2 is a completely novel virus and there was no vaccine available in March 2020, the entire populations of WA and CO were assumed to be susceptible to SARS-CoV-2 infection. Thus, the number of susceptible 
individuals in either state on March 15th, $S_{0}$, was initially estimated as:

$$
\begin{aligned}
& N=S_{0}+E_{0}+A_{0}+Q_{0}+I_{0}+R_{0}+D_{0} \\
& N-E_{0}-A_{0}-Q_{0}-I_{0}-R_{0}-D_{0}=S_{0}
\end{aligned}
$$

Here, $\mathrm{N}$ is the total population size of the state and the other variables are the initial values for the given state's SEAQIRD compartments. Importantly, equation 9 only defined the initial estimate of $S_{0}$, but $S_{0}$ was allowed to vary during the model fitting process (see section 3.6).

Since COVID-19 mortality data is generally reliable, the number cumulative deaths up until March 15 th, $D_{0}$, was not adjusted in the raw data. On the other hand, estimating the number of symptomatic, infected individuals required some adjustment. Ideally, the initial number of I individuals $\left(I_{0}\right)$ could be calculated as:

$$
I_{0}=C_{0}-D_{0}-R_{0}
$$

Where $C_{0}, D_{0}$, and $R_{0}$ are cumulative numbers of active COVID-19 cases, deaths, and recoveries up until March 15th, respectively. For simplicity, $R_{0}$ and $H_{0}$ were assumed to be small relative to $C_{0}$ and $D_{0}$ early in the pandemic, leaving just:

$$
I_{0}=C_{0}-D_{0}
$$

However, $C_{0}$ is drastically underestimated for the US population. One preprint study suggests that increasing the number of cases in the US by 179 percent would bring the US death rate down to a similar rate as South Korea, a country with reliable infection counts, after accounting for demographic differences [17]. Thus, $I_{0}$ was estimated as:

$$
I_{0}=2.79 C_{0}-D_{0}
$$

This calculation assumes that the correction for the US applies to individual states as well. The initial number of exposed individuals $\left(E_{0}\right)$ and asymptomatic infected individuals $\left(A_{0}\right)$ was assumed to be directly proportional to $I_{0}$. In the absence of reliable data on these numbers, we decided:

$$
\begin{gathered}
E_{0}=30 I_{0} \\
A_{0}=8 I_{0}
\end{gathered}
$$

because these numbers generated biologically reasonable model curves and they reflect how the majority of COVID-19 transmission occurs through asymptomatic individuals [19].

Finally, the initial number of symptomatic, isolated individuals $\left(Q_{0}\right)$ was assumed to be: 


$$
Q_{0}=10 H(0)
$$

Here, $H(0)$ is the number of individuals hospitalized with COVID-19 on March 15th. In other words, for every individual hospitalized with COVID19, there were assumed to be nine other symptomatic individuals that were self-isolating at home.

\subsection{Sensitivity analysis}

Once we developed the baseline SEAQIRD models $\left(E_{0}=30^{*} I_{0}, Q_{0}=10^{*} H(0)\right.$, $p=0.86, q=0.986$, and $\chi=1)$ for $\mathrm{CO}$ and WA conditions and parameter values were chosen, we tested the sensitivity of the WA and CO SEAQIRD models in two ways. First, we used the sensFun() command in the FME package to analyze the local sensitivity of the SEAQIRD model to all of its initial conditions and parameters [35]. This analysis was repeated twice, once for WA initial conditions (see Figures S2A, S2B, and S3) and once for CO initial conditions (see Figures $\mathrm{S} 6 \mathrm{~A}, \mathrm{~S} 6 \mathrm{~B}$, and S7). Second, we then focused on the parameters $p$ and $q$ for both SEAQIRD models since they had by far the most influence on the model output. We re-ran both the WA and CO models under different values of $p$ and $q$ values while holding all other initial conditions at their baseline values. Estimates of $p$ vary widely in published literature, so we ran the SEAQIRD model with two different values of $p(0.86$ and 0.425$)$ while holding all other parameters and conditions at their baseline values (see Figures S9-S12). The first value of $\mathrm{p}$ comes from a study that estimated the fraction of cases that went undocumented during a period of COVID-19 spread in China [19]. Cases often go undocumented when infected individuals have symptoms that are too mild for them to be concerned with getting tested for infection. Thus, we reasoned this fraction should be close to the value of $p$. The second value of $\mathrm{p}$ comes from a census in Italy where the authors directly observed 42.5 percent of their COVID-19 infected participants lacking symptoms [18]. We also ran the SEQIRD model with two different values of $q$, its baseline value of 0.986 and a value of 0.972 (see Figures S13 - S16), which doubles the probability of dying from a symptomatic infection relative to the baseline SEAQIRD model.

\subsection{Fitting SEAQIRD to data}

We used the Levenberg-Marquardt algorithm implemented in the FME package to optimize $\alpha$ and $S_{0}$ such that the sum of squared error between the SEAQIRD model output and the curve of cumulative deaths over time for either WA or $\mathrm{CO}$ was minimized. We only fit these two parameters because these are the parameters that have the most influence on the model's results besides $p$ and $q$ (see Figures S2 and S7). However, unlike $p$ and $q$ these numbers could not be reasonably estimated from previous studies. Furthermore, we could not fit additional parameters with the paucity of data points we had without overfitting. Finally, we also only chose to fit the SEAQIRD model to mortality data because 
estimates of other state variables, such as symptomatic infections, are probably massively under-counted. In all model fitting cases, the initial estimate of $S_{0}$ was given by equation 9 and the initial estimate of $\alpha$ was $3 \mathrm{E}-7$, but these values were allowed to vary within bounds during the fitting process. $\alpha$ was always bounded between $3 \mathrm{E}-6$ and $3 \mathrm{E}-8$ while $S_{0}$ was bounded between 0.05 and 1.5 times its initial estimate. These bounds were chosen because they produced biologically reasonable results (see Figures 2, S1A, and S5A).

\subsection{Estimating probability of hospitalization given symp- tomatic infection}

The number of individuals hospitalized with COVID-19 was assumed to be a constant fraction of the number of individuals in compartment $\mathrm{Q}$ (see equation 8). After simulating the SEAQIRD model based on the initial conditions described above, this fraction was estimated for each state model by plotting the number of hospitalizations in the state against the number of quarantined individuals output by the fitted SEAQIRD model at each time point. The slope of the ordinary least squares regression line through these points (see Figures S1B and S5B) estimated the probability of hospitalization given symptomatic infection $(h)$, since all quarantined individuals are either hospitalized or not.This resulted in an obvious underestimation of $h$ (see Figures S1C and S5C). Thus, we also tried estimating $h$ numerically by multiplying the $\mathrm{H}(\mathrm{t})$ function by 10000 different constants increasing from 0 to 0.1 in increments of $1 \mathrm{E}-5$ and calculating the sum of squared error between the resulting curve and hospitalization counts over time. The constant that gave the lowest sum of squared error between $H(t) * h$ and the curve of hospitalization counts over time, after correcting for under-reporting, thus estimated $h$ (see Figures 5A and 5B).

\subsection{Estimating effect of decreased hospitalization and in- creased bed cap on delaying bed shortage}

Pre-pandemic surveys suggest that about 50 - 80 percent of ICU beds in America are typically occupied at any given time [43]. Thus, we assumed that 30 percent of ICU beds in either WA or CO could be reasonably allocated to COVID-19 infected patients during the modeling period, which is referred to as the hospital "bed cap" at some points in the paper. Once the probability of hospitalization given symptomatic infection (hereto referred to as the "hospitalization probability") was estimated from the fit of SEAQIRD model to the hospitalization

data, we tested the effect of altering the probability of hospitalization and the bed cap simultaneously by plugging 10000 different combinations of these two numbers spanning values from $0-1$, in increments of $1 \mathrm{E}-5$, into the SEAQIRD model (see Figures S4, S8, S10, S12, S14, S16). 


\section{Results}

\subsection{Effective population sizes for COVID-19 transmission}

Figure 2 presents the optimized baseline SEAQIRD model's fits to mortality data for CO and WA. The best fit parameters for $\alpha$ and $S_{0}$ in under these conditions were 5.990521e-07 and 4.814597e+05, respectively, for the WA SEAQIRD model and 4.687143e-07 and 7.675917e+05, respectively, for the CO SEAQIRD model (see Figure 2). The curves for the other state variables in the SEAQIRD model, suggest that WA and CO had 39,805 and 53,742 symptomatic infected individuals $(\mathrm{I}+\mathrm{Q})$, respectively, at their peak infection periods between March 15th and May 18th (see Figures S1 and S5). The number of asymptomatic infected cases $(\mathrm{E}+\mathrm{A})$, on the other hand, peaked at 280,280 and 388,234 individuals for WA and CO respectively during this period. In the SEAQIRD model, the peak number of hospitalizations occurred 24 and 37 days after March 15th for WA an CO, respectively. The actual peak in hospitalizations for these states during the modeling period, however, occurred 21 days and 39 days after March 15th, respectively. The probability of hospitalization given symptomatic infection was estimated as 0.0290 (see Figure 3A) and 0.0236 (see Figure 3B) for WA and CO, respectively. During the modeling period, CO started out as having fewer death cases than WA, but then overtook WA death counts by the end of the modeling period.

\subsection{Effect of decreasing hospitalization on delaying bed shortage}

Hospitalizing fewer COVID-19 patients delayed the time at which the bed capacity for COVID-19 patients was exceeded (Figure 4). This effect increased as fewer COVID-19 patients were hospitalized, according to the concave up shape of the graph in Figure 4. The curves for both WA and CO reach asymptotes; in other words, values of the hospitalization probability below which the bed cap will never be exceeded. This occurred around a probability of 0.0123 for WA and about 0.00567 for $\mathrm{CO}$.

\subsection{Effect of increasing bed cap on delaying bed shortage}

The graph of how the number of days until the bed cap is exceeded based on the proportion of available ICU beds looks markedly different between WA and CO (Figure 5). The curve for CO initially shows a concave down shape, but inflects around a value of of 0.5. The curve for WA, on the other hand, has a mostly concave up shape. Both curves approach an asymptote (see dotted lines in Figure 5) as the bed cap increases. For WA, this asymptote occurred around a bed cap of 57.5 percent of the maximum. The asymptote for $\mathrm{CO}$, on the other hand, occurred at a bed cap of about 77.6 percent of the maximum. For a non-zero proportion of bed availability, bed caps were always exceeded later in $\mathrm{CO}$ than in WA. 


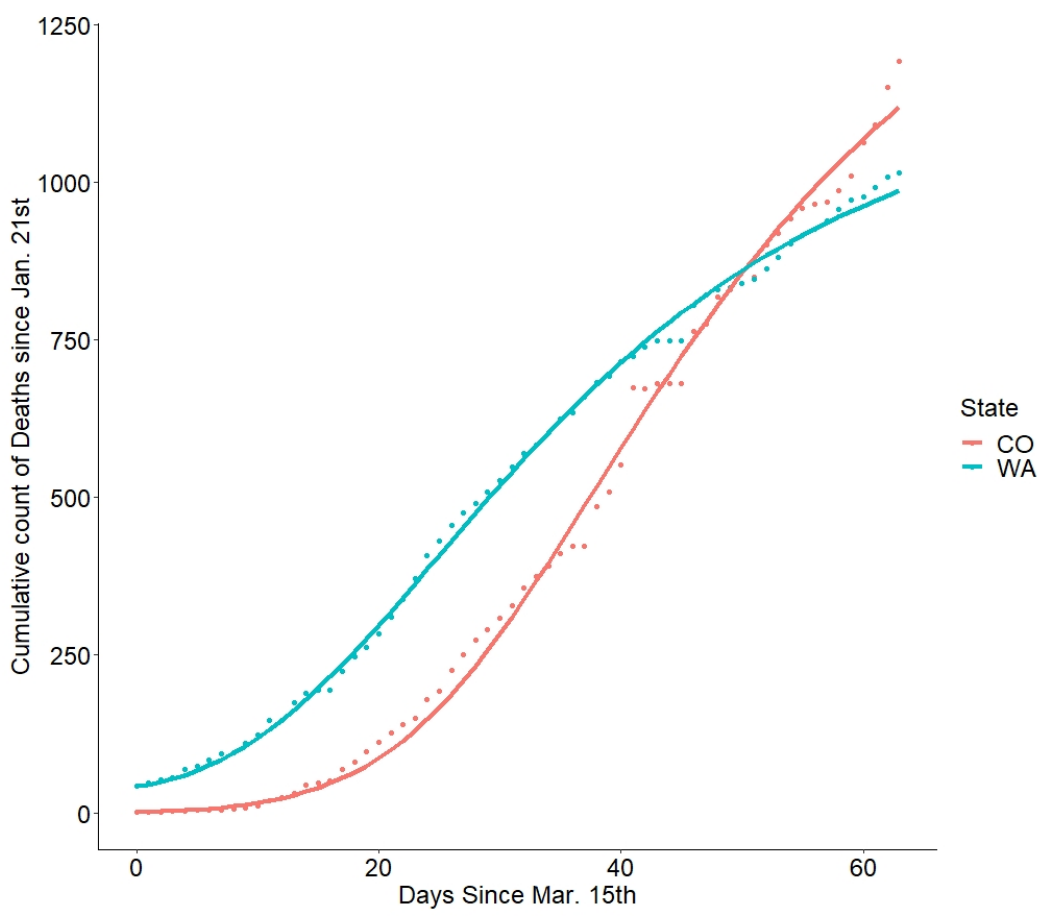

Figure 2: Points are raw counts of deaths due to COVID-19 since Jan. 21st - colored by the state in which the deaths occurred - and the lines are the SEAQIRD model fits to these points. For both models $E_{0}=30^{*} I_{0}, Q_{0}=$ $10^{*} H(0), p=0.86, q=0.986$, and $\chi=1$.

\subsection{SEAQIRD sensitivity to conditions and parameters}

Once the SEAQIRD model was fit to each state's data, we tested the local sensitivity of the SEAQIRD model to its initial conditions and $\alpha$. We first used the local sensitivity analysis functions implemented in the FME package to identify the initial conditions with the largest influence on model output. $S_{0}$, $\alpha$, and $D_{0}$, are well-estimated either directly from data or from the model fitting, whereas the values of the remaining conditions were assumed. Thus, we took the two parameters the SEAQIRD model was most sensitive to, $p$ and $q$, altered their values, and recorded the effect on the SEAQIRD model's predictions. We ran the SEAQIRD model with $p$ set to 0.86 and 0.425 , while keeping all other parameters and initial conditions at their baseline values. We also tried setting $q$ to either 0.986 (it's value in the baseline model) or 0.972 (which doubles the probability of dying from infection). The different values for $p$ gave very different curves for both the WA and CO SEAQIRD models (see Figures S9 and S11), as did the different values of $q$ (see Figures S13 and S15). However, even in these alternative scenarios, the effects of altering the hospitalization probability retained a more concave upward shape than altering the bed cap (see Figures 

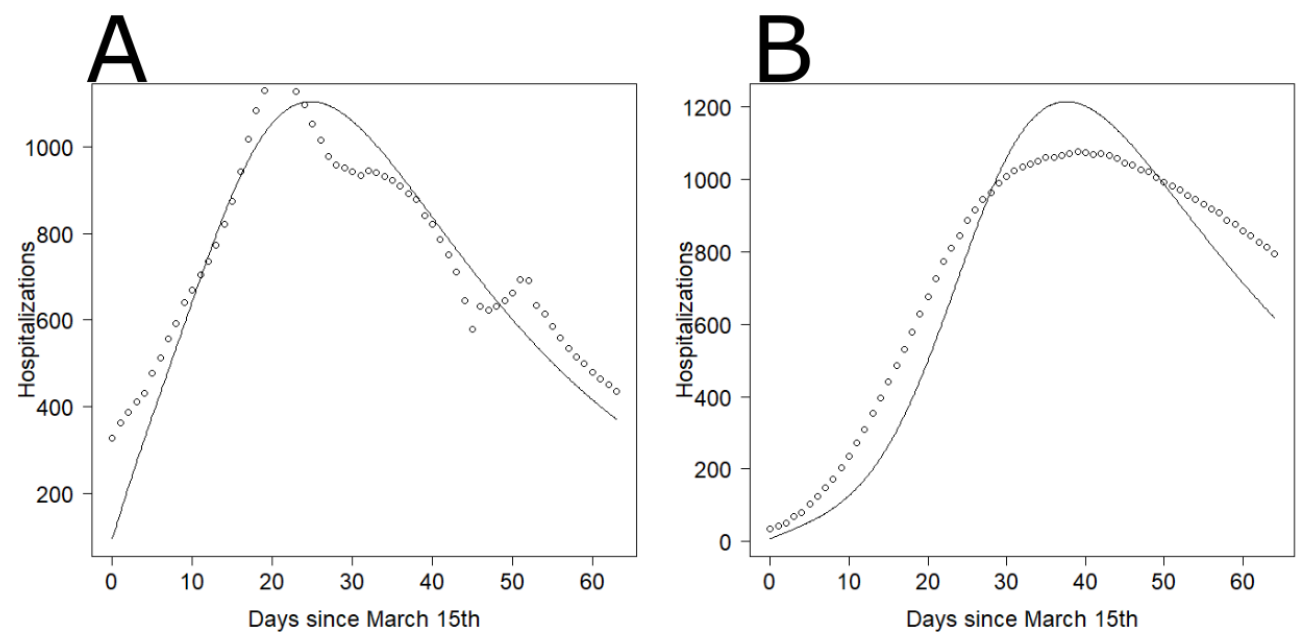

Figure 3: (A)Points are counts of hospitalized COVID-19 patients in all WA hospitals by day, corrected for underreporting by hospitals. Curve is $\mathrm{Q}(\mathrm{t})$ function output from SEAQIRD model multiplied by the probability of hospitalization given symptomatic infection, which was numerically estimated as 0.0289829 . (B) Points are counts of hospitalized COVID-19 patients in all CO hospitals by day, corrected for underreporting by hospitals. Curve is $\mathrm{Q}(\mathrm{t})$ function output from SEAQIRD model multiplied by the probability of hospitalization given symptomatic infection, which was estimated to be about 0.02357236 . For both (A) and (B), SEAQIRD initial conditions included $E_{0}=30^{*} I_{0}, Q_{0}=10^{*} H(0)$, $p 0=0.86, q=0.986$ and $\chi=1$.

S10, S12, S14, S16).

\section{Discussion}

Some of SARS-CoV-2's defining characteristics are that a large fraction of infected individuals do not develop symptoms and that there's a significant presymptomatic period during which an individual can still spread the virus. This has led to massive levels of undocumented infection; some pre-print articles estimate that 19 million Americans have caught COVID-19, only a small fraction of which are laboratory confirmed cases [22]. We developed a novel system of differential equations to account for these asymptomatic and presymptomatic phases of COVID-19 infection, which happened to be similar to other models used to model COVID-19 spread [22]. This system was fit to data on COVID19 mortality, the most reliable data available on COVID-19 spread, in two US states. These different states had different infection dynamics and different peak infection dates. Including infection peaks in the analysis was especially important because deterministic models like SEAQIRD can be especially misleading 


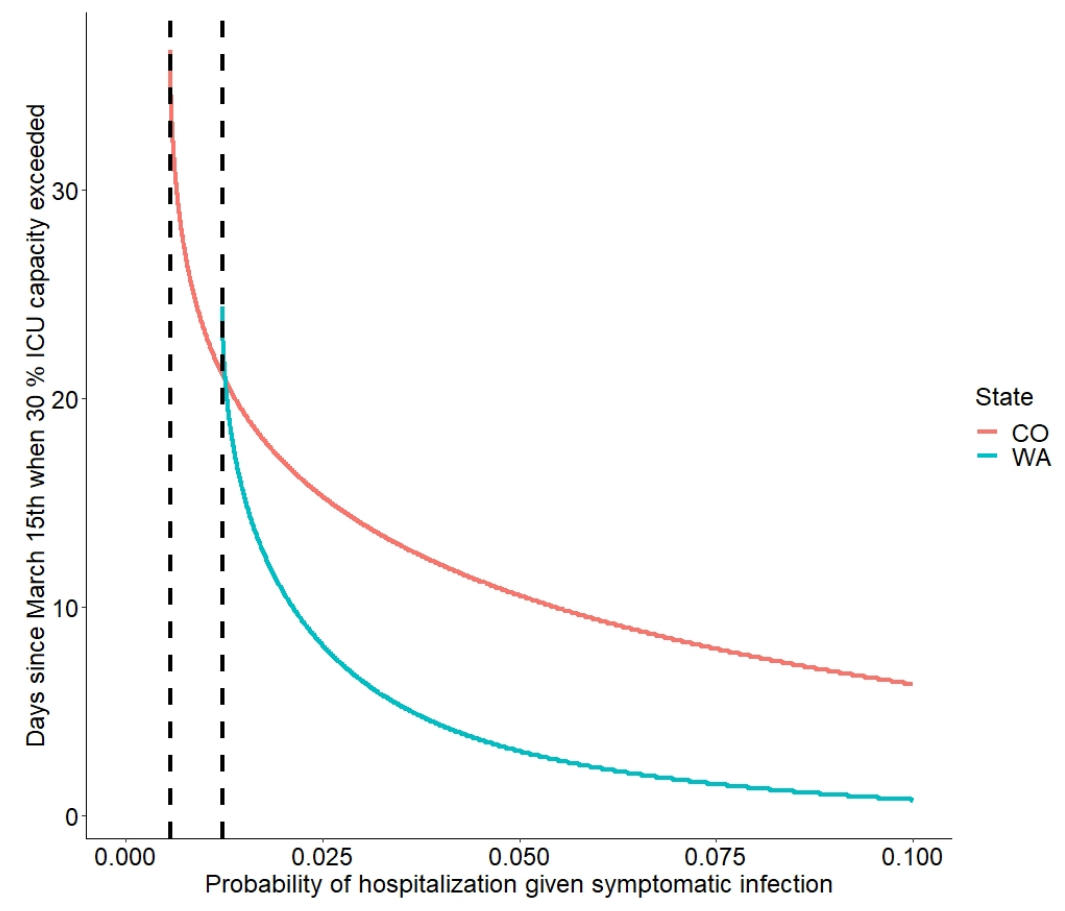

Figure 4: Dotted vertical lines represent asymptotes - the probability beyond which the ICU bed cap would not be exceeded from March 15th - May 18th. Both models used their baseline values for initial conditions and parameters.

when fit with only pre-peak data [10]. We then compared the effects of decreasing the probability of hospitalization given symptomatic infection and increasing the proportion of ICU beds available to COVID-19 patients on delaying when the number of hospitalized COVID-19 patients exceeded the allotted ICU bed capacity. Under the conditions simulated here, we observed that when the probability of hospitalization is already low, decreasing it further can have a large effect on delaying an ICU bed shortage. Meanwhile, altering the proportion of ICU beds available to COVID-19 patients had different effects on when a bed shortage was reached in the two states. This finding was very robust for both the WA and CO models under multiple sets of initial conditions and parameter values.

\subsection{Altering bed cap has different effects on delaying a bed shortage in WA and CO}

Our analyses focused on two parameters of the SEAQIRD model that best represented potential interventions for preventing a hospital bed shortage: the number of beds available to COVID-19 patients (expressed as a proportion of all 


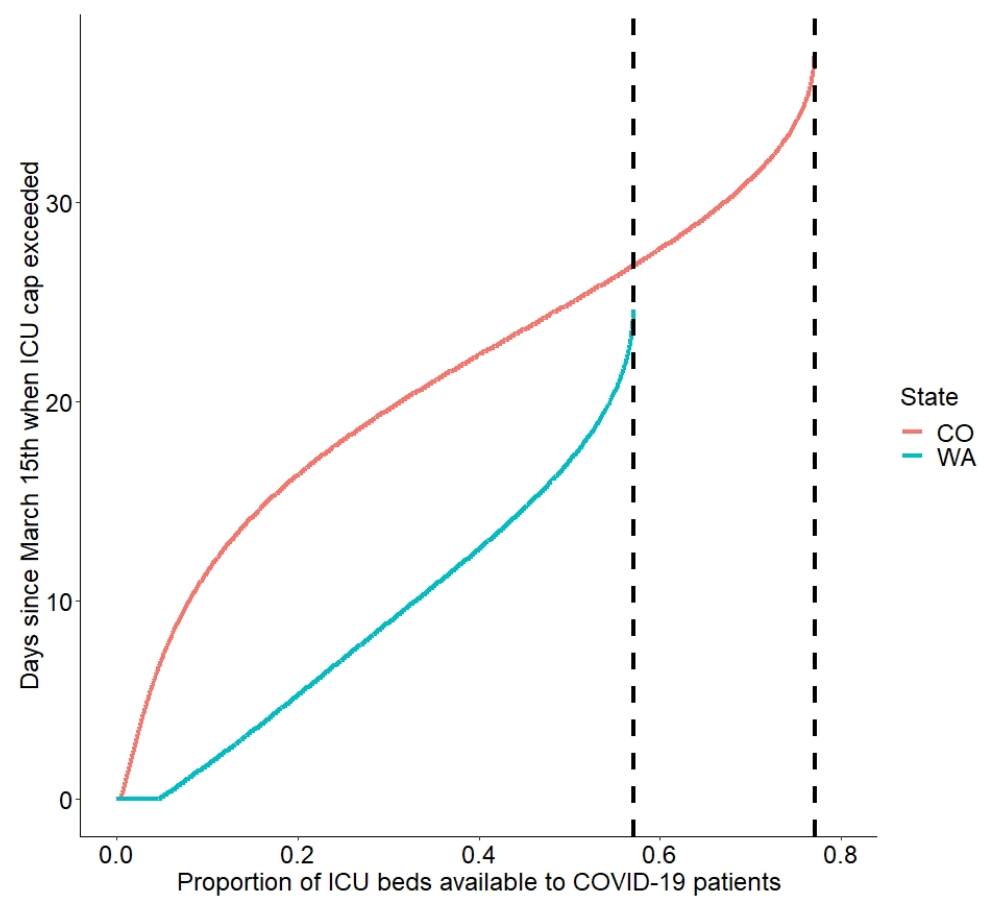

Figure 5: Dotted vertical lines represent asymptotes - proportion of ICU bed availability beyond which the ICU bed cap would not be exceeded from March 15 th - May 18th . For both models $E_{0}=30^{*} I_{0}, Q_{0}=10^{*} H(0), p=0.86, q=$ $0.986, \chi=1$.

beds in the state) and the probability of a symptomatic, infected individual of being hospitalized $(h)$. We altered both of these parameters and observed their effects on when an ICU bed shortage (defined as the number of hospitalized individuals exceeding the number of available beds) was reached in WA and CO.

First, we altered one parameter while holding the other constant. For both WA and $\mathrm{CO}$, decreasing the hospitalization probability had larger effects on delaying a bed shortage if this probability was already small (see Figure 4). However, the effect of increasing the bed cap differed drastically between the WA and CO models. Increasing the bed cap has a more sigmoid relationship with delaying a bed shortage in CO than it does in WA (see Figure 5). This is potentially due to differences in the total number of cases relative to hospital beds in both states. The maximum number of infected individuals $(\mathrm{I}+\mathrm{A})$ was much higher in CO than in WA $(53,742$ vs 39,805$)$ while the number of ICU beds was also much lower in CO than WA (973 vs 1564). These factors combined could have made increasing the number of ICU beds in CO initially less effective at halting a bed shortage, leading to the sigmoid relationship in 
Figure 5. We also altered both the hospitalization probability and bed cap at the same time and observed similar results (see Figures S10, S12, S14, S16). Overall, our findings do not conflict with current CDC guidelines that tell sick individuals to not go to hospitals unless they are experiencing severe COVID-19 symptoms [40].

\subsection{SEAQIRD predictions are robust to initial conditions and parameter values}

The parameters with by far the largest influence on SEAQIRD dynamics are the probability of developing a symptomatic infection given COVID-19 exposure $(p)$ and the probability of dying from a symptomatic COVID-19 infection $(q)$. Early studies on COVID-19 suggested that $p$ could be fairly low - around 20 percent or less [16], [26]. Later studies suggested that this probability is likely higher - upwards of 50 percent [18],[19]. Changing the values of $p$ and $q$ in the SEAQIRD model had a large influence on the model output relative to the other parameters and initial conditions (see Figures S2A, S3, S6A, S7). Thus, we suspect that changes in $p$ and $q$ over time or between different groups of people are mainly responsible for the poor fits between the SEAQIRD model and hospitalization data (see Figure 3A and 3B). Rewriting these parameters to be dependent on demographic characteristics, such as age, could improve these fits.

\subsection{Low effective population size for COVID-19 transmis- sion in $\mathrm{CO}$ and WA}

The effective population size of both the WA and CO SEAQIRD models was orders of magnitude lower than the actual population sizes of these states as estimated by the US census. This discrepancy is partly due to the lack of any lockdown effects in the SEAQIRD model. Thus, COVID-19 was restricted to spreading among only a subset of the WA and CO populations. The lack of lockdown effects is arguably SEAQIRD model's most obviously violated assumption. WA lockdowns began on March 23rd 2020 [15], not long into the simulation, closely followed by $\mathrm{CO}$ on March 26th [23]. However, there is some pre-print work suggesting that US individuals were already relaxing physical distancing measures by mid-April, perhaps limiting the lockdown effect in our data [44]. Other phenomenon could have also contributed to the small effective population size in the SEAQIRD model. For example, there are well-documented cases of individuals unexposed to COVID-19 having antibodies that react to SARS-CoV2 particles [32]. There's also population-level evidence suggesting that certain pre-existing vaccines may have trained immune systems against COVID-19, although these vaccines are not common in the US [5],[12]. Nonetheless, it is possible that pre-existing and trained immunity may have removed individuals from the susceptible populations in CO and WA, explaining why COVID-19 only spread among a small subset of these populations in the SEAQIRD model. Incorporating lockdown effects, pre-existing immunity, and trained immunity 
into SEAQIRD-like models may improve their predictions. Understanding how these nuances affect hospitalizations will be incredibly important as the US continues to control the spread of COVID-19 and any variants that arise.

\section{References}

[1] E. Abdollahi, M. Haworth-Brockman, and Y. Keynan, et al., Simulating the effect of school closure during COVID-19 outbreaks in Ontario, Canada. BMC Med., 18 (2020), pp. 2-8.

[2] N. Aguirre-Duarte, Can people with asymptomatic or pre-symptomatic COVID-19 infect others: a systematic review of primary data. medRxiv, 2020.04.08.20054023, 2020.

[3] C. Anastassopoulou, L. Russo, and A. Tsakris, et al., Data-based analysis, modelling and forecasting of the COVID-19 outbreak. PLoS ONE, 15, e0230405.

[4] M. Bellisle and R. La Corte, Washington scrambles for hospital beds, https://www.columbian.com/news/2020/mar/19/washington-scrambles-forhospital-beds/ (19 March 2020)

[5] B. Brook, D. J. Harbeson, and C. P. Shannon, et al., BCG vaccination-induced emergency granulopoiesis provides rapid protection from neonatal sepsis, Sci. Transl. Med., 12. (2020), eaax4517.

[6] S. K. Brooks, R. K. Webster, and L. E. Smith, et al., The psychological impact of quarantine and how to reduce it: rapid review of the evidence. The Lancet, 395 (2020), pp. 912-920.

[7] A. Chandrashekar, J. Liu, and A. J. Martinot, et al., SARS-CoV-2 infection protects against rechallenge in rhesus macaques Science, 369 (2020), pp. 812817.

[8] S.Andrew, The psychology behind why some people won't wear masks https://www.cnn.com/2020/05/06/health/why-people-dont-wear-maskswellness-trnd/index.html. (6 May 2020).

[9] C. Courtemanche, J. Garuccio, and A. Le, et al., Strong Social Distancing Measures In The United States Reduced The COVID-19 Growth Rate. H. Aff. Mill., 39 (2020), pp. 1237-1246.

[10] J. Daunizeau, R. J. Moran, and J. Mattout, et al., On the reliability of model-based predictions in the context of the current COVID epidemic event: impact of outbreak peak phase and data paucity, preprint, medRxiv, 2020.04.24.20078485, 2020.

[11] A. van Dorn, R. E. Cooney, and M. L. Sabin, COVID-19 exacerbating inequalities in the US Lancet Lond. Engl., 395 (2020), pp. 1243-1244. 
[12] L. E. Escobar, A. Molina-Cruz, and C. Barillas-Mury, BCG vaccine protection from severe coronavirus disease 2019 (COVID-19), Proc. Natl. Acad. Sci. U. S. A., 117 (2020), pp17720-17726.

[13] D. Graeupner and A. Coman, The dark side of meaning-making: How social exclusion leads to superstitious thinking. J. Exp. Soc. Psychol., 69 (2017), pp. 218-222.

[14] D. Guan, D. Wang, and S. Hallegatte, et al., Global supply-chain effects of COVID-19 control measures, Nat. Hum. Behav., 4 (2020), pp. 577-587.

[15] J. Inslee, Inslee announces "Stay Home, Stay Healthy" order, https://www.governor.wa.gov/news-media/inslee-announces-stay-homestay-healthy

[16] J. R. Koo, A. R. Cook, and M. Park, et al., Interventions to mitigate early spread of SARS-CoV-2 in Singapore: a modelling study, Lancet Infect. Dis., 20 (2020), pp. 678-688.

[17] A. Lachmann, Correcting under-reported COVID-19 case numbers, preprint, medRxiv 2020.03.14.20036178, 2020.

[18] E. Lavezzo, E. Franchin, and C. Ciavarella, et al., Suppression of a SARSCoV-2 outbreak in the Italian municipality of Vo' Nature, 584 (2020), pp. 425-429.

[19] R. Li, S. Pei, and B. Chen, et al., Substantial undocumented infection facilitates the rapid dissemination of novel coronavirus (SARS-CoV-2), Science, 368 (2020), pp. 489-493.

[20] K. Liu, Y. Chen, and R. Lin, et al., Clinical features of COVID-19 in elderly patients: A comparison with young and middle-aged patients, J. Infect., 80 (2020), pp. e14-e18.

[21] Q.-X. Long, X.-J. Tang, and Q.-L. Shi, et al., Clinical and immunological assessment of asymptomatic SARS-CoV-2 infections, Nat. Med., 26 (2020), pp. 1200-1204.

[22] A. Mahajan, R. Solanki, and N. Sivadas, Estimation of Undetected Symptomatic and Asymptomatic cases of COVID-19 Infection and prediction of its spread in USA, medRxiv 2020.06.21.20136580, 2020.

[23] K. Marshall, G. M. Vahey, and E. McDonald, et al., Exposures Before Issuance of Stay-at-Home Orders Among Persons with Laboratory-Confirmed COVID-19 - Colorado, March 2020. Morb. Mortal. Wkly. Rep., 69, pp. $847-849$.

[24] C. S. Martarelli, and W. Wolff, Too bored to bother? Boredom as a potential threat to the efficacy of pandemic containment measures, preprint, PsyArXiv/s41599-020-0512-6, 2020. 
[25] T. M. McMichael, D. W. Currie, and S. Clark, et al., Epidemiology of Covid19 in a Long-Term Care Facility in King County, Washington, N. Engl. J. Med., 382 (2020), pp. 2005-2011.

[26] K. Mizumoto, K. Kagaya, and A. Zarebski, et al., Estimating the asymptomatic proportion of coronavirus disease 2019 (COVID-19) cases on board the Diamond Princess cruise ship, Yokohama, Japan, 2020, Euro Surveill., 25 (2020), 22.

[27] S. M. Moghadas, A. Shoukat, and M. C. Fitzpatrick, et al., Projecting hospital utilization during the COVID-19 outbreaks in the United States, Proc. Natl. Acad. Sci., 117 (2020), pp. 9122-9126

[28] M. Mossa-Basha, J. Medverd, and K. Linnau, et al., Policies and Guidelines for COVID-19 Preparedness: Experiences from the University of Washington. Radiology, (2020), 201326.

[29] S. C. Newbold, D. Finnoff, and L. Thunström, et al., Effects of Physical Distancing to Control COVID-19 on Public Health, the Economy, and the Environment, Environ. Resour. Econ., (2020), pp. 1-25.

[30] I. J. Ramírez and J. Lee, COVID-19 Emergence and Social and Health Determinants in Colorado: A Rapid Spatial Analysis Int. J. Environ. Res. Public. Health, 17 (2020), 3856.

[31] R Core Team, $R$ : A language and environment for statistical computing. $R$ Foundation for Statistical Computing, https://www.R-project.org/ (2020).

[32] A. Sette and S. Crotty, Pre-existing immunity to SARS-CoV-2: the knowns and unknowns, Nat. Rev. Immunol., 20 (2020), pp. 457-458.

[33] A. Shoukat, C. R. Wells, and J. M. Langley, et al., Projecting demand for critical care beds during COVID-19 outbreaks in Canada, CMAJ, 192 (2020), pp. e489-e496.

[34] M. J. Siedner, G. Harling, and Z. Reynolds, et al., Social distancing to slow the US COVID-19 epidemic: Longitudinal pretest-posttest comparison group study, PLoS Med., 17 (2020), e1003244.

[35] K. Soetaert and T. Petzoldt, Inverse Modelling, Sensitivity and Monte Carlo Analysis in R Using Package FME, J. Stat. Softw., 33 (2010), pp. $1-28$.

[36] K. Soetaert, T. Petzoldt, and R. W. Setzer, Solving Differential Equations in R: Package deSolve, J. Stat. Softw., 33 (2010), pp. 1-25.

[37] D.B.G. Tai, A. Shah, and C. A. Doubeni, et al. The Disproportionate Impact of COVID-19 on Racial and Ethnic Minorities in the United States, Clin. Infect. Dis., (2020), pp. 1-4 
[38] S. Y. Tartof, L. Qian, and V. Hong, et al., Obesity and Mortality Among Patients Diagnosed With COVID-19: Results From an Integrated Health Care Organization, Ann. Intern. Med., (2020), pp. 1-10.

[39] R. Verity, L. C. Okell, and I. Dorigatti, et al., Estimates of the severity of coronavirus disease 2019: a model-based analysis, Lancet Infect. Dis., 20 (2020), pp. 669-677.

[40] Center for Disease Control and Prevention (CDC) What to Do If You Are Sick, https://www.cdc.gov/coronavirus/2019-ncov/if-you-are-sick/stepswhen-sick.html (15 August 2020).

[41] H. Wickham, ggplot2: Elegant Graphics for Data Analysis, Springer-Verlag Inc., New York, NY, 2016.

[42] J. T. Wu, K. Leung, and M. Bushman, et al., Estimating clinical severity of COVID-19 from the transmission dynamics in Wuhan, China, Nat. Med., 26 (2020), pp. 506-510.

[43] H. Wunsch, J. Wagner, and M. Herlim, ICU Occupancy and mechanical ventilator use in the United States, Crit. Care Med., 41 (2013), pp. 27122719 .

[44] J. Zhao, M. Lee, and S. Ghader, et al., Quarantine Fatigue: first-ever decrease in social distancing measures after the COVID-19 outbreak before reopening United States, preprint, arXiv:2006.03716 [cs], 2020. 